\title{
The Cause of Azimuthal Variations in Directional Reflectance of Vegetative Canopies
}

\author{
GWYNN H. SUITS \\ Willow Run Laboratories, The University of Michigan, Ann Arbor, Michigan
}

\begin{abstract}
The variation of the directional reflectance of a vegetative canopy with azimuthal view angle becomes prominent when the canopy is illuminated by the sun at large angles from zenith. An extension of a previous directional reflectance model of vegetative canopies is presented to quantify this effect. The results indicate that the cause of the azimuthal variation can be traced to solar flux illumination of the vertically oriented canopy components and that the extreme variations of reflectance with azimuth of view are moderated by the azimuthally isotropic sources of flux from skylight and canopy.
\end{abstract}

\section{Introduction}

The success of the airborne multispectral scanning technique in taking inventory of the type and distribution of vegetative canopies by automatic pattern recognition processing depends upon the existence of a regular relationship between received spectra and the canopy type. The spectral reflectance of vegetative canopies governs the nature of the received spectra in the spectral ranges from the ultraviolet through the near infrared. However, the spectral reflectance of a canopy depends upon more than just the structure and biological content of the canopy. It also depends upon the angle of illumination and on both polar and azimuthal angles of view. The magnitude, and consequently the value, of the azimuthal reflectance variation to remote sensing tasks is often overlooked.

In a previous paper, Suits (1972) derived the relationship between the biological content and structure of a canopy and the directional reflectance variations due to sun angle and polar angle of view. A special reflectance variation with azimuthal and polar angles near the subsolar point, frequently referred to as the "hot spot," was also explained on the basis of the dependence on the probabilities of line of sight for specular flux and of line of sight for viewing.

In this paper an expression for additional azimuthal variation is derived. This azimuthal variation differs fundamentally from the hot spot in that it is caused by the azimuthal variations in radiance from each elemental layer of the canopy. It can be observed at all nonzero polar angles and is most evident in canopies with vertical and nearly opaque components illuminated at large polar sun angles and viewed from large polar viewing angles.

The azimuthal variation is illustrated in the photographs shown in Figs. 1(a) and (b). These photographs were taken with an early morning sun (polar angle $77^{\circ}$ ) with a fairly clear sky condition in January. The subject being photographed is a spot in an Ann Arbor, Michigan, public park. Each photograph shows the same spot viewed at a large polar viewing angle but at different azimuthal directions relative to the sun. Figure 1(a) is for an azimuth of $0^{\circ}$ relative to the sun (the sun at the observer's back). Figure 1(b) is for an azimuth angle of $180^{\circ}$. The camera settings were unchanged.

The park grass canopy is bounded at the bottom by a light snow. Notice that the snow appears to be about the same brightness in each photograph but that the brightness of the grass changes markedly. The small tree and the guard posts show what can be expected of completely opaque vertical objects. The shadow back side of these objects are illuminated by sky light and diffuse flux from the canopy. The means by which this effect may be represented mathematically in an extension of the previous directional reflectance model is shown in the following paragraphs.

\section{Summary of Model Concepts and Nomenclature}

The model concepts and nomenclature are first summarized in order to place the extension of the 


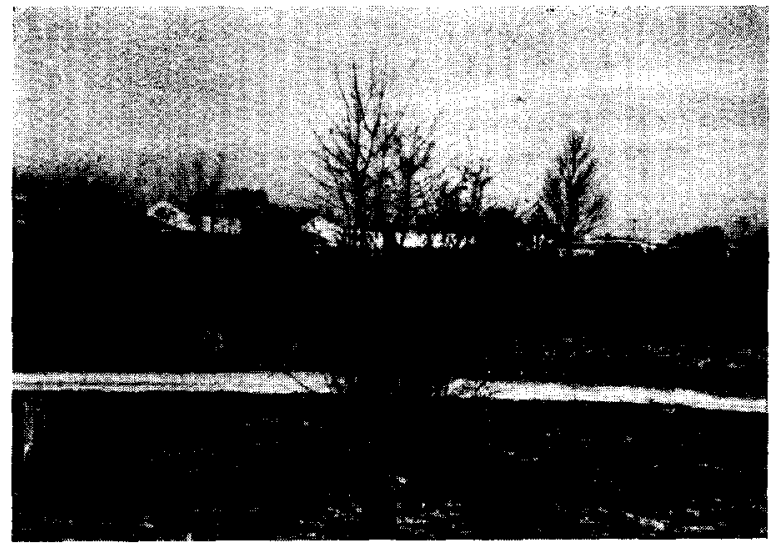

(a)

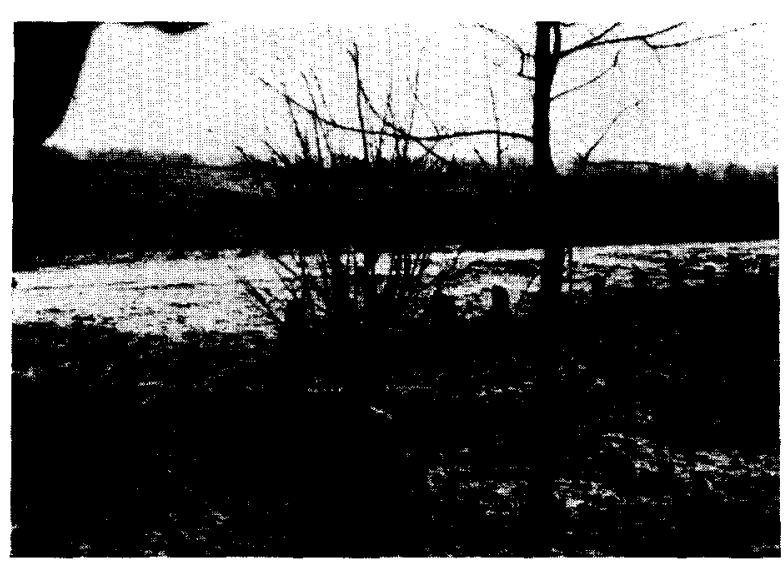

(b)

FIG. 1. Azimuthal variation of canopy reflectance. These photographs of the ground are taken with polar sun angle, $77^{\circ}$, and polar view angle, $\sim 65^{\circ}$, at a series of azimuthal angles, $\psi$, relative to the solar flux direction. a is for $\psi=0^{\circ}$ (sun at observer's back) and $\mathrm{b}$ is for $\psi=180^{\circ}$. Camera settings are unchanged. A remote sensor will not resolve the details but will respond to the sum of the contributions from each part.

canopy model in proper context. The canopy model idealizes a canopy in two ways. First, the canopy is assumed to consist of several layers containing distinct mixtures of biological components. Second, the biological components are idealized by replacing each biological component by three flat Lambertian plane sections found by taking the three mutually orthogonal projections of each component. The spectral qualities of Lambertian plane sections are derived directly from those of the biological component which they represent. These layers, along with the ensemble of horizontal and vertical projections of components within the layers, describe the mathematical model.
Now, with methods initiated by Kubelka and Munk (1931) and extended by Duntley (1942), the radiant flux field within the canopy is determined. Three types of radiant flux are identified, the upward directed, diffuse, spectral flux density, $E_{\lambda}(+d)$, the downward directed, diffuse, spectral flux density, $E_{\lambda}(-d)$, and the downward, specular, spectral flux density directed along polar angle $\theta$, $E_{\lambda}(s, \theta)$. The specular flux is that flux from an outside source, such as the sun, which has not yet been intercepted by any component in its flow into the canopy. The diffuse flux is that flux which has been scattered at least once by a canopy component.

This flux field within the canopy is the source of illumination of the components within the canopy. Thus, the radiance of each infinitesimal layer of components may be calculated as was shown in the previous paper, Suits (1972). The contributions to the infinitesimal layer spectral radiance, $\Delta L_{\lambda}$, of the azimuthally isotropic diffuse flux is easily seen to be

$$
\begin{aligned}
\Delta L_{\lambda}(+d)= & {\left[\sigma_{h} n_{h} \Delta x \tau+\sigma_{v} n_{v} \Delta x(2 / \pi)\right.} \\
& \times \tan \phi(\tau / 2+\rho / 2)] E_{\lambda}(+d) / \pi
\end{aligned}
$$

for upward diffuse flux, and

$$
\begin{aligned}
\Delta L_{\lambda}(-d)= & {\left[\sigma_{h} n_{h} \Delta x \rho+\sigma_{v} n_{v} \Delta x(2 / \pi)\right.} \\
& \times \tan \phi(\tau / 2+\rho / 2)] E_{\lambda}(-d) / \pi
\end{aligned}
$$

for downward diffuse flux where

$\sigma_{h}$ is the average horizontal projection area for a component,

$\sigma_{v}$ is the average vertical projection area for a component,

$n_{h}$ is the number of horizontal projections per unit volume,

$n_{v}$ is the number of vertical projections per unit volume,

$\Delta x$ is the thickness of the infinitesimal layer being illuminated,

$\tau$ is the hemispherical spectral transmittance of the component,

$\rho$ is the hemispherical spectral reflectance of the component,

$\phi$ is the polar angle of view.

The first term on the right in relation (1) is the radiance due to illumination from below which is diffusely transmitted through the horizontal components. The first term on the right in relation 
(2) is the radiance due to the diffuse reflection of downward diffuse flux by horizontal components.

The second term in both relation (1) and (2) is the radiance due to oblique rays of diffuse flux which are intercepted by the vertical components. Half of the diffuse flux strikes obliquely from behind the vertical component and is transmitted towards the viewer through the component while the other half of the diffuse flux strikes the front of the vertical component and is reflected towards the viewer. The factor $(2 / \pi)$ is the azimuthal average of the fractional area of the flat vertical component in the direction of view when the vertical components are assumed to have surface normals randomly oriented in azimuth. The factor, $\tan \phi$, accounts both for the projected area of vertical components as seen from polar angle $\phi$ as well as for the increased oblique line of sight through the infinitesimal layer.

Because the diffuse flux field is azimuthally isotropic and the normals to vertical components are randomly oriented in azimuth, no contribution to an azimuthal variation in infinitesimal layer radiance can be expected from the diffuse flux field. The azimuthal variation in radiance is due entirely to the contribution of the specular flux field.

In the previous paper, the azimuthal contribution of specular flux to the canopy radiance was avoided by the simple expedient of employing an azimuthal average.

Using the azimuthal average, the contribution of specular flux was

$$
\begin{aligned}
\Delta L_{\lambda}(s)= & {\left[\sigma_{h} n_{h} \rho+\sigma_{v} n_{v}(2 / \pi)^{2}(\tau / 2+\rho / 2)\right.} \\
& \times \tan \phi \tan \theta] E_{\lambda}(s) / \pi,
\end{aligned}
$$

which is entirely appropriate for azimuthally symmetrical sources.

The radiance of each infinitesimal layer was then calculated by adding the diffuse and specular contributions,

$$
\Delta L_{\lambda}=u E_{\lambda}(+d) / \pi+v E_{\lambda}(-d) / \pi+w E_{\lambda}(s) / \pi
$$

where $u, v$, and $w$ represent the corresponding right hand factors in relations (1)-(3). Most remote sensing systems such as aerial photography and multispectral scanners using reflected daylight are traditionally operated so as to view nearly straight down at such times of day when the sun is near zenith. Under such conditions the azimuthal variation of canopy reflectance is often small compared to the polar angle variation and often remains unobservable. However, if the information needed for canopy identification is obtainable from the azimuthal variation of canopy reflectance then oblique sun angles and oblique view angles may well be the optimum conditions for a remote sensing mission even though the oblique sun angles provide lower flux levels and oblique viewing produces images that are far from the traditional map format. The calculation of the azimuthal variation is a worthy task.

\section{The Specular Flux Contribution as a Function of Aximuth}

When the horizontal components of the canopy intercept specular flux, the layer radiance due to specular flux is seen by reflection from the upper surface of the component. Since the components were assumed to be Lambertian, no azimuthal variation in radiance can arise from such interception.

The vertical components, on the other hand, will be seen by transmission or reflection depending upon the azimuthal position of the observer relative to the sun. If the sun is at the observer's back (zero azimuth relative to the sun) all vertical components will contribute radiance by the diffuse reflection of specular flux; whereas, if the observer faces the direction of the sun $\left(180^{\circ}\right.$ azimuth relative to the sun), all vertical components will contribute radiance by the diffuse transmission of specular flux. These extreme cases occur even though the normals to the surfaces of vertical components are randomly oriented in azimuth. If the observer views the canopy at azimuth angles relative to the sun between $0^{\circ}$ and $180^{\circ}$, then some mixture of reflection and transmission contributions due to specular flux will be seen. In order to determine what this mixture should be, consider the following derivation.

Let the azimuthal direction from component to sun be the zero azimuth. Let the direction of normal to the vertical component be $\psi_{n}$ and positive in the clockwise direction. Let the azimuthal direction from component to view position be $\psi$ and positive in the clockwise direction.

The viewer will see reflected specular flux when $\psi-\pi / 2 \leqslant \psi_{n} \leqslant \pi / 2$ as shown in Fig. 2 and 


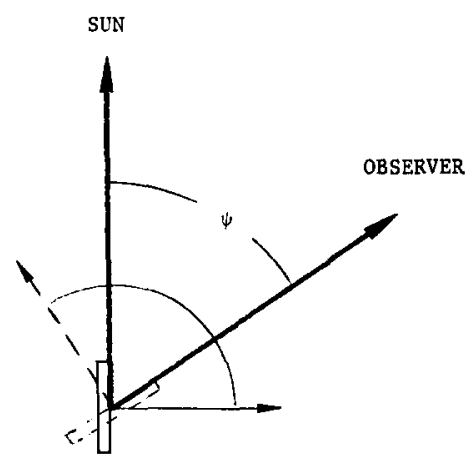

FIG. 2. Conditions for viewing reflection. Reflected specular flux will be seen by an observer at azimuth, $\psi$, provided that the normals to vertical components lie within the azimuthal range shown.

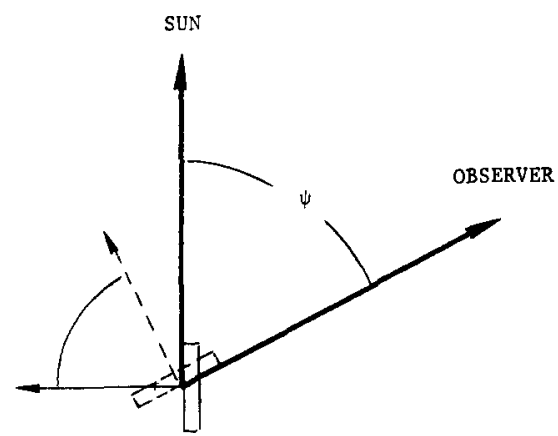

FIG. 3. Conditions for viewing transmission. Transmitted specular flux will be seen by an observer at azimuth, $\psi$, provided that the normals to vertical components lie within the azimuthal range shown.

will see transmitted specular flux when $-\pi / 2 \leqslant$ $\psi_{n} \leqslant \psi-\pi / 2$ as shown in Fig. 3 .

The flux intercepted, $\Phi$, by a vertical component is

$$
\Phi=\sigma_{v} \cos \psi_{n} \sin \theta[E(s) \sec \theta],
$$

where

$\sigma_{v}$ is the area of the vertical component,

$E(s)$ is the irradiance of specular flux on a horizontal plane,

$\theta \quad$ is polar angle of specular flux.

The radiant intensity for reflection in the direction of component normal is then

$$
I(\rho)=\rho \sigma_{v} \cos \psi_{n} \tan \theta E(s) / \pi,
$$

and the component radiates in Lambertian fashion. For transmission, similarly,

$$
I(\tau)=\tau \sigma_{v} \cos \psi_{n} \tan \theta E(s) / \pi .
$$

The intensity in the direction of view is proportional to the projected area of the vertical component, thus,

$$
\begin{aligned}
I(\text { as seen } \phi, \psi)= & (\rho \text { or } \tau) \sigma_{v} \cos \psi_{n} \tan \theta[E(s) / \pi] \\
& \times \cos \left(\psi-\psi_{n}\right) \sin \phi .
\end{aligned}
$$

The radiance of a layer $\Delta x$ thick with $n_{v}$ such components per unit volume is then

$$
\begin{gathered}
\Delta L=(\rho \text { or } \tau) \sigma_{v} n_{v}(\Delta x \sec \phi) \cos \psi_{n} \tan \theta \\
\times \cos \left(\psi-\psi_{n}\right) \sin \phi[E(s) / \pi] .
\end{gathered}
$$

Now one must find the average radiance when reflection is viewed at angle $\psi$ and when transmission is viewed at angle $\psi$. Every vertical component is seen by either one or the other but not both. The sum of these will be the total first surface interaction contribution to infinitesimal layer radiance due to specular flux.

The average radiance at angle $\psi$ over random component orientations for reflection is proportional to

$$
\frac{1}{\pi-\psi} \int_{\psi-\pi / 2}^{\pi / 2} \cos \psi_{n} \cos \left(\psi-\psi_{n}\right) \mathrm{d} \psi_{n},
$$

and for transmission

$$
\frac{1}{\psi} \int_{-\pi / 2}^{\psi-\pi / 2} \cos \psi_{n} \cos \left(\psi-\psi_{n}\right) \mathrm{d} \psi .
$$

Using trigonometric identities lavishly and integrating, one obtains the reflectance contribution average

$$
\frac{1}{2}\left[\cos \psi+\frac{\sin \psi}{\pi-\psi}\right],
$$

and for transmittance,

$$
\frac{1}{2}\left[\frac{\sin \psi}{\psi}-\cos \psi\right] .
$$

The azimuthal variation factors, [ $\cos \psi+\sin \psi$ ] $(\pi-\psi)]$ and $[\sin \psi / \psi-\cos \psi]$ are mirror images about the value $\psi=\pi / 2$. The factor for reflectance is shown in Fig. 4.

Since the horizontal component is Lambertian the contribution, $\Delta L_{\lambda}(s)$, due to specular flux becomes

$$
\begin{aligned}
\Delta L_{\lambda}(s)= & \left\{\sigma_{h} n_{h} \rho_{h}+\sigma_{v} n_{v} \frac{\tan \phi \tan \theta}{2}\right. \\
& \times\left[\rho_{v}\left(\cos \psi+\frac{\sin \psi}{\pi-\psi}\right)+\cdots\right. \\
& \left.\left.\quad+\tau_{v}\left(\frac{\sin \psi}{\psi}-\cos \psi\right)\right]\right\} E_{\lambda}(s) / \pi .
\end{aligned}
$$




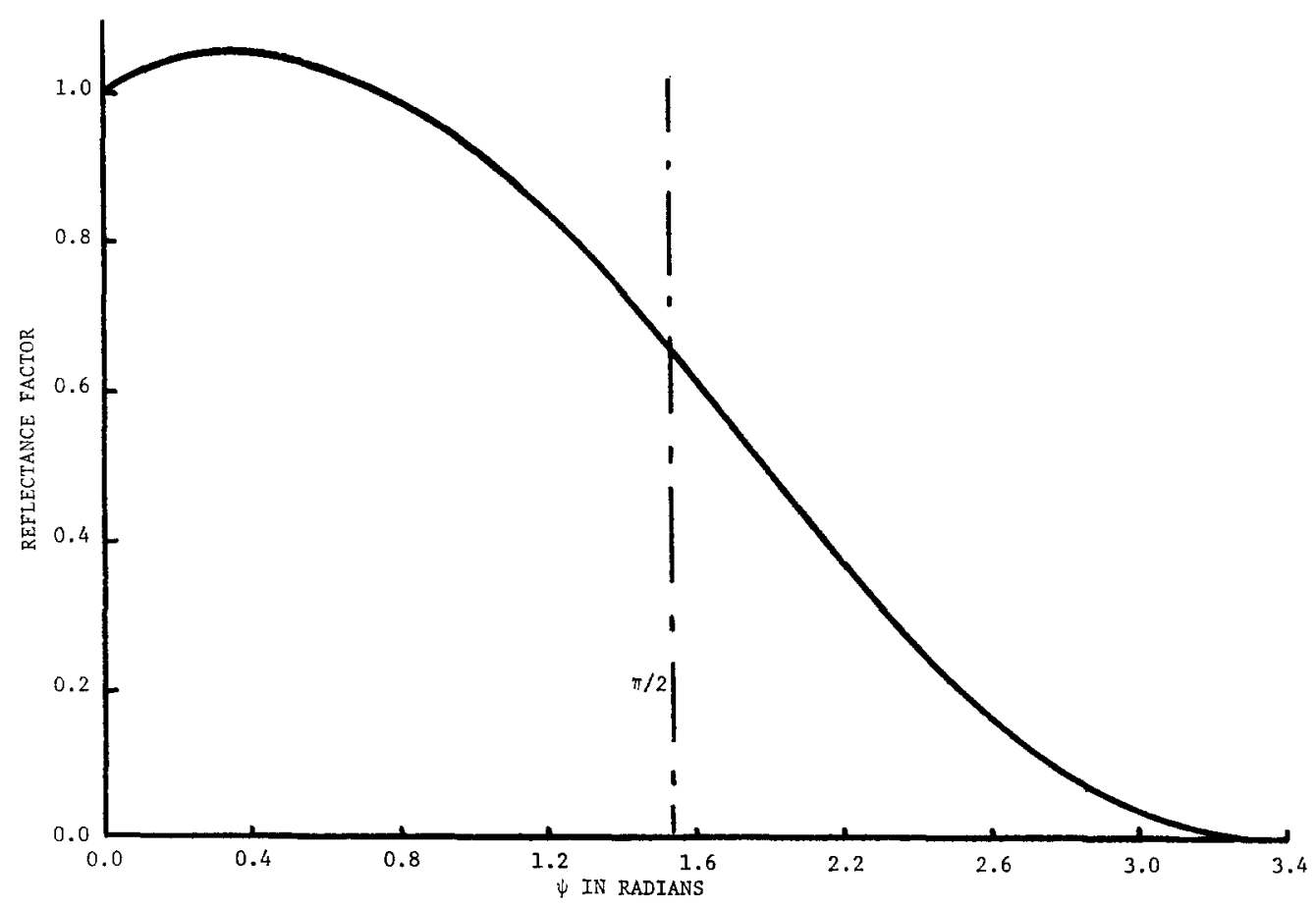

FIG. 4. Form of the reflectance factor. The curve is proportional to the weighted average contribution to radiance due to reflection of specular flux from vertical components as a function of observer azimuth, $\psi$. The curve for transmission is a mirror image of the reflectance factor about the line $\pi / 2$.

Thus,

$$
\Delta L_{\lambda}(s)=w^{\prime} E_{\lambda}(s) / \pi
$$

where

$$
\begin{aligned}
& w^{\prime}=\sigma_{h} n_{h} \rho_{h}+\sigma_{v} n_{v} \tan \phi \tan \theta\left[\left(\rho_{v} / 2\right)\right. \\
& \times\left(\cos \psi+\frac{\sin \psi}{\pi-\psi}\right)+\cdots \\
&\left.+\left(\tau_{v} / 2\right)\left(\frac{\sin \psi}{\psi}-\cos \psi\right)\right] .
\end{aligned}
$$

The infinitesimal layer radiance including the azimuthal variation becomes

$$
\Delta L_{\lambda}=u E_{\lambda}(+d) / \pi+v E_{\lambda}(-d) / \pi+w^{\prime} E_{\lambda}(s) / \pi .
$$

The canopy radiance can now be calculated exactly as it was done in the previous paper. In the final formula for directional reflectance $w^{\prime}$ replaces $w$.

When the specular flux contribution to the canopy radiance is much greater than the diffuse flux contribution, the directional reflectance in a single layer canopy of depth, $l$, is given by

$$
\begin{aligned}
\pi L_{\lambda} / E_{\lambda} \cong & w^{\prime}(1-\exp [-l(K+k)]) /(K+k)+\cdots \\
& +\exp [-l(K+k)] \\
& \times \rho(\text { soil }),
\end{aligned}
$$

where

$$
\begin{aligned}
& k=\sigma_{h} n_{h}+(2 / \pi) \sigma_{v} n_{v} \tan \theta, \\
& K=[\left.\sigma_{h} n_{h}+(2 / \pi) \sigma_{v} n_{v} \tan \phi\right]\{1-\exp \\
&\left.\times\left[-\beta(\theta-\phi)^{2}-\gamma \psi^{2}\right]\right\} .
\end{aligned}
$$

The two quantities, $\beta$ and $\gamma$, are correlation constants which are measures of the fineness of texture of the canopy producing the hot spot effect.

For very large polar view angles such that $l(k+K) \gg 1$, relation (8) simplifies still more to

$$
\begin{gathered}
\pi L_{\lambda} / E_{\lambda} \cong\left(\frac{1}{2}\right) \tan \theta\left[\rho\left(\cos \psi-\frac{\sin \psi}{\pi-\psi}\right)\right. \\
\left.+\tau\left(\frac{\sin \psi}{\psi}-\cos \psi\right)\right],
\end{gathered}
$$

provided that the vertical leaf area index is not excessively small.

\section{Discussion}

It is easy to see in relation (9) that the canopy reflectance changes from component transmittance dominant to component reflectance dominant with azimuth of view and, furthermore, that an increase in sun angle, $\theta$, and an increase in the 
difference between $\rho$ and $\tau$ also increases this azimuthal variation.

It is interesting to find that the leaf area indices do not appear in relation (9). Large polar angles of view are common for ground based observation. Such view angles make one particularly insensitive to the amount of biomass in a canopy. No change in azimuth angle will improve matters. However, if one wishes to distinguish fields by component spectral type independently of the degree of biomass, then a large polar angle of view is particularly advantageous. Two separate reflectance measurements, one at $\psi=0^{\circ}$ and one at $\psi=180^{\circ}$, will yield values largely dependent upon component reflectance and transmittance respectively.

A canopy composed of opaque components which are moderately high in reflectance should produce very marked azimuthal variations. Indeed, according to relation (9), the reflectance of the canopy should reach zero at $\psi=180^{\circ}$. The fact that the reflectance never reaches zero in nature is explained by the fact that two other contributions are usually present, the azimuthally symmetric skylight and the azimuthally symmetric diffuse flux field of the canopy. Consider the following cases. If the sun is near zenith, the solar specular flux is much greater than skylight but the value of $\tan \theta$ is small so the azimuthal variation is reduced. If the polar sun angle is large, the irradiance of sunlight on a horizontal plane is reduced and becomes comparable with skylight so that skylight moderates the azimuthal variation. In the blue spectral band, skylight contributes significantly in proportion to sun light at most solar angles. In the infrared spectral range where clear skylight contributes little flux, green vegetation produces copious diffuse flux so the azimuthal variation is reduced by diffuse flux. In the red spectral range where both diffuse skylight and diffuse canopy flux are low, the transmittance and reflectance of green vegetation are nearly equal and have very low values so that again the azimuthal variation is reduced. Therefore, it is not surprising that extreme azimuthal variations are not often found in canopies of green vegetation.

To illustrate what could be expected of a canopy devoid of leaves, consider a hypothetical deciduous stand of trees in the winter time on a snow covered soil. A calculation of the directional reflectance was made of such a stand using the geometric data from a single young tree 8-m high. The reflectance of the bark was considered to be 0.20 and the reflectance of the snow 0.90 . The stand was taken to have 0.08 trees of this kind per square meter. For such a plant density the crowns of the trees would overlap frequently.

Fig. 5 displays the azimuthal variation of directional reflectance for four different situations

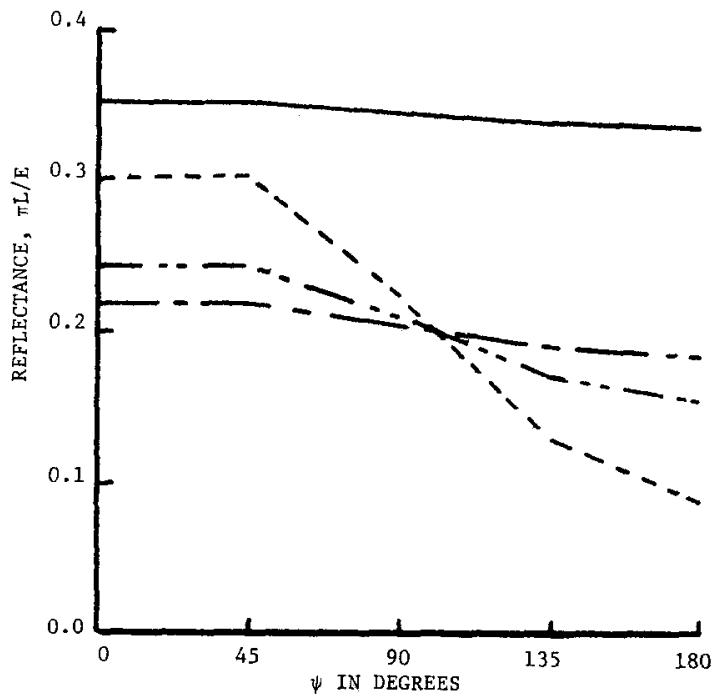

FIG. 5. Reflectance of winter deciduous forest. The azimuthal variation of the reflectance of a hypothetical winter defoliated forest on snow soil is shown for combinations of sun angle, $\theta$, and polar view angle, $\phi$. Skylight is missing. The solid curve is for $\theta=25^{\circ}, \phi=50^{\circ}$; dash-dot curve is for $\theta=25^{\circ}, \phi=75^{\circ}$. Notice that the high sun does not produce a strong azimuthal variation. The dash-dotdot curve is for $\theta=75^{\circ}, \phi=50^{\circ}$; the dotted curve is for $\theta=75^{\circ}, \phi=75^{\circ}$. The azimuthal variation is clearly brought out by the conditions for the dotted curve. The hot spot conditions would occur at the $\psi=0^{\circ}$ point on the dotted curve. The hot spot increase is not shown here.

- a high sun $\left(\theta=25^{\circ}\right)$ with two polar view angles $\left(\phi=50^{\circ}\right.$ and $\left.75^{\circ}\right)$, and a low sun $\left(\theta=75^{\circ}\right)$ with the same pair of polar view angles. Skylight is considered to be completely absent. Notice that the high sun case yields only minor azimuthal variation while the change of polar view angle is quite large in comparison. The low sun angle case also shows a polar view angle variation but the azimuthal variation is now a dominant factor. The extreme variation occurs for $\theta=75^{\circ}$ and $\phi=75^{\circ}$ with a canopy reflectance of 0.30 at $\psi=0^{\circ}$ down to 0.09 at $\psi=180^{\circ}$. These extremes are about a factor of three apart. One can expect that the azimuthally independent snow covered soil helps to moderate these extremes. 
If the snow were missing and were replaced by a soil with reflectance 0.05 , one would find the azimuthal variations shown in Fig. 6. The

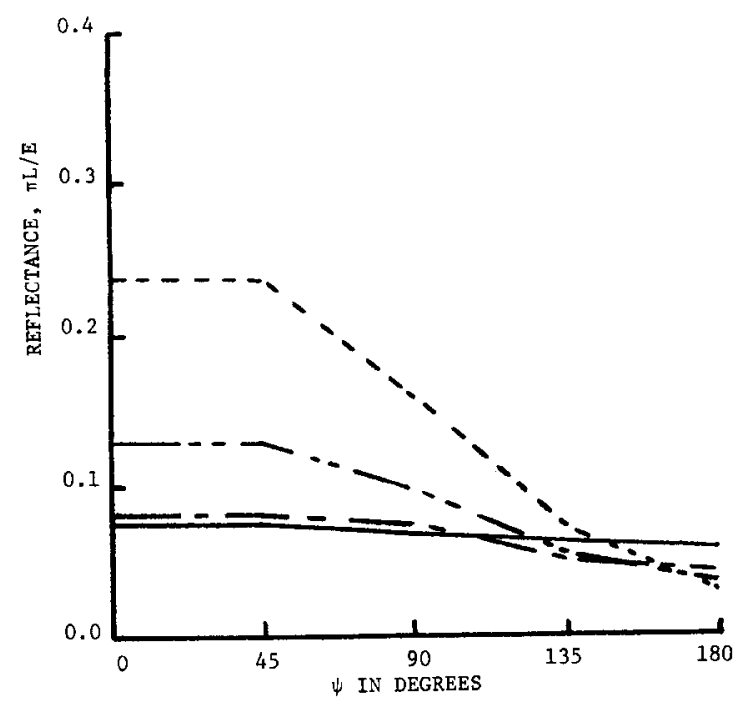

FIG. 6. Reflectance of defoliated forest on dark soil. The same conditions were used here as were used for the reflectance curves for Fig. 5 except that the soil reflectance was made 0.05 instead of 0.90 . This situation could arise after an early spring thaw. Solid curve is for $\theta=25^{\circ}, \phi=50^{\circ}$; dash-dot curve is for $\theta=25^{\circ}, \phi=75^{\circ}$; dash-dot-dot curve is for $\theta=75^{\circ}, \phi=50^{\circ}$; dotted curve is for $\theta=75^{\circ}, \phi=75^{\circ}$. The skylight is still missing. Notice that a high sun still yields little azimuthal variation but the low sun produces dramatic variation.

greatest azimuthal variation occurs for the same conditions on sun and view angles as before but the extremes are now at 0.24 and 0.03 , about a factor of eight apart. There is little significant diffuse flux within the canopy under these conditions. The low reflectance value, 0.03 , is largely due to line of sight to the soil through the canopy.

The high value of reflectance at $\psi=0^{\circ}$ appears to be contradictory at first glance. The average value of component reflectance, 0.20 for bark and 0.05 for soil, can hardly exceed 0.20 . The higher value of 0.24 stems from the definition of canopy reflectance-that is, $\pi \mathrm{L} / \mathrm{E}$, where the irradiance, $\mathrm{E}$, is the irradiance on a horizontal plane and not the irradiance on a plane normal to the solar flux. If the irradiance on a plane normal to the solar flux is $E$ (normal), the irradiance on a horizontal plane is $E$ (normal) $\cos \theta$. Thus, as the sun sets, the irradiance on a horizontal plane approaches zero while the vertical parts of the canopy still intercept some solar flux which is scattered in the direction of view. The radiance remains finite. In this way, reflectance values can increase to infinity.

The moderating influence of the two sources of diffuse flux is illustrated in Fig. 7. The case of

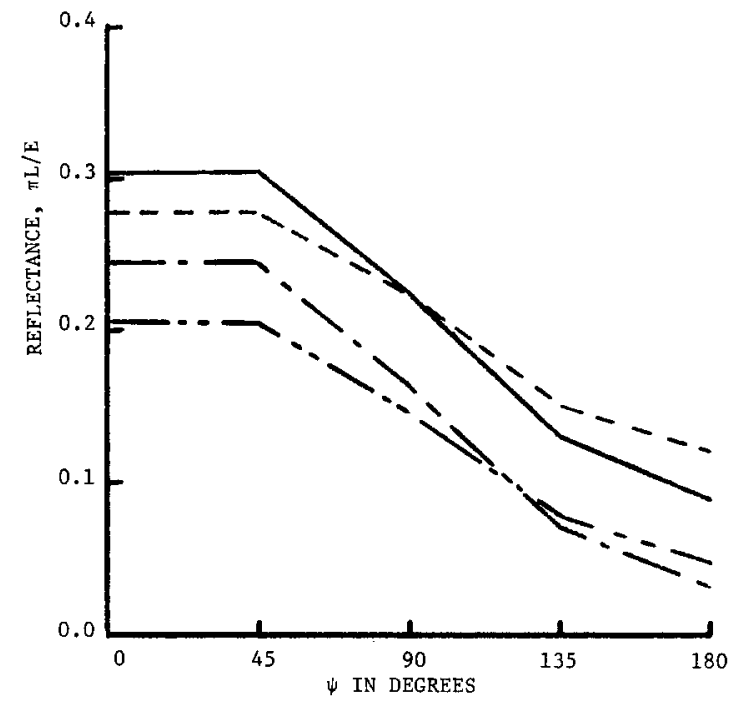

FIG. 7. Moderating effects of diffuse flux. The azimuthal variation for the case, $\theta=75^{\circ}, \phi=75^{\circ}$ is shown with and without the diffuse flux contributions. Solid curve, snow cover without skylight; dash-dot curve no snow cover, no skylight; dash-dot-dot curve, no snow but with skylight; dotted curve, both snow and skylight.

low sun angle, $\theta=75^{\circ}$, and low view angle, $\phi=75^{\circ}$, is taken along with the assumption that diffuse skylight contributes one fourth of the irradiance on a horizontal plane while the sun contributes three fourths. Four cases are shownthe dark soil without skylight, the dark soil with skylight, the light soil (snow) without skylight, and the light soil with skylight. The introduction of both skylight and light soil brings the extreme values for azimuthal variation to about a factor of two as compared to the factor of eight for the case where both sources of diffuse flux are missing. The shapes of these curves are similar to that of the azimuthal variation factor curve shown in Fig. 4. Since the only source of azimuthal variation is traceable to this function-the transmittance being zero-the resemblance is expected.

\section{Summary}

A previous directional reflectance model of a vegetative canopy has been extended to include the general azimuthal variation in canopy reflect- 
ance. The cause of the azimuthal variation is the azimuthal variation of the observed proportions of reflection and transmission of the specular flux field by the vertical components of the canopy. The interaction of the canopy components with the diffuse flux field and skylight, along with reflection of specular flux from horizontal components, tends to moderate the azimuthal variations.

The natural conditions found with canopies of green vegetation under daylight tend to yield less azimuthal variation than do canopies of defoliated deciduous forests.

When azimuthal variations in reflectance are large for large polar view angles, the canopy reflectance at zero azimuth is dominated by vertical component reflectance and at $180^{\circ}$ azimuth by vertical component transmittance independent of the biomass of the canopy.

The author wishes to thank Professor James T. Wilson, Director of the Institute of Science and Technology, The University of Michigan, for his support of this work.

\section{References}

Duntley, S. (1942) J. Opt. Soc. Am. 32, 61.

Kubelka, P. and F. Munk (1931) Z., Tech. Physik 11, 593. Suits, G. H. (1972) Remote Sensing of Environment 2, 117. 